\title{
Prognostic value of the combination of GRACE risk score and mean platelet volume to lymphocyte count ratio in patients with ST-segment elevation myocardial infarction after percutaneous coronary intervention
}

\author{
XINSEN CHEN $^{1 *}$, MENG SHAO $^{2 *}$, TIAN ZHANG ${ }^{1}$, WEI ZHANG ${ }^{1}$, \\ YOUBAO MENG ${ }^{1}$, HONGYAN ZHANG $^{1}$, HUA HAI $^{1}$ and GUIHUA LI ${ }^{1}$ \\ Departments of ${ }^{1}$ Emergency and ${ }^{2}$ Pathophysiology, The First Affiliated Hospital, \\ Shihezi University School of Medicine, Shihezi, Xinjiang 832002, P.R. China
}

Received May 11, 2019; Accepted December 17, 2019

DOI: $10.3892 / \mathrm{etm} .2020 .8626$

\begin{abstract}
The Global Registry of Acute Coronary Events (GRACE) risk score and the mean platelet volume to lymphocyte count ratio (MPVLR) can be used independently to predict adverse outcomes in patients with acute coronary syndromes. However, the level of MPVLR in relation to the GRACE score, and whether a combination of these methods can better predict the clinical adverse outcome of patients with ST-segment elevation myocardial infarction (STEMI), have not been previously examined. Therefore, the aim of the present study was to investigate whether the combination of GRACE risk score and MPVLR is a good predictor of a 30-day major adverse cardiovascular events (MACE) in patients with STEMI. A total of 464 patients with STEMI undergoing percutaneous coronary intervention (PCI) were enrolled, and divided into four groups based on the optimal cut-off values for GRACE score and MPVLR. GRACE score and MPVLR levels were separately recorded during admission. Spearman's rank correlation analysis showed a positive correlation between GRACE score and MPVLR ( $\mathrm{Q}=0.304 ; \mathrm{P}<0.001)$. Both GR ACE score [hazard ratio (HR), 1.706; 95\% CI, 1.435-3.058; $\mathrm{P}<0.001]$ and MPVLR level (HR, 1.668; 95\% CI, 1.202-2.170; $\mathrm{P}<0.001$ ) were found to be independent predictors of a 30-day MACE. Additionally, the
\end{abstract}

Correspondence to: Professor Guihua Li, Department of Emergency, The First Affiliated Hospital, Shihezi University School of Medicine, 107 North Second Road, Shihezi, Xinjiang 832002, P.R. China

E-mail: lgh169@126.com

${ }^{*}$ Contributed equally

Key words: ST-segment elevation myocardial infarction, the Global Registry of Acute Coronary Events risk score, mean platelet volume to lymphocyte ratio, prognosis high MPVLR + high GRACE score group of patients had an HR of 2.455 (95\% CI, 1.736-3.188) for a 30-day MACE, when using the low MPVLR + low GRACE score group as a reference. Based on the area under the curve, MPVLR combined with GRACE scores achieved an improved performance in differentiating angiographic no-reflow during a 30-day MACE, compared with individual MPVLR and GRACE scores. Therefore, the present results suggested that the GRACE score may be positively correlated with MPVLR and that their combination accurately predicted the occurrence of short-term MACE in patients with STEMI after PCI.

\section{Introduction}

As the world's ageing population increases, ST-segment elevation myocardial infarction (STEMI) incidence is expected to increase to be the most common and fatal cardiac emergency (1). Acute myocardial infarction (AMI) is often caused by the interruption of coronary artery blood flow and myocardial ischemic necrosis resulting from decreased stability of coronary atherosclerotic plaque, ulcer, rupture and other thrombosis (2). Therefore, early, rapid and complete opening of the infarcted artery is key to improving the prognosis of patients with STEMI $(2,3)$. Percutaneous coronary intervention (PCI) is a non-surgical method used in extensive myocardial reperfusion therapy (4). Early risk stratification and identification of high-risk patients with STEMI are of great significance in prognosis, and also in guiding diagnosis and treatment decisions (5). Currently, the Global Registry of Acute Coronary Events (GRACE) score is widely used as an acute risk stratification tool in the evaluation of prognosis in patients with acute coronary syndrome (ACS) (6). GRACE score parameters include age, systolic blood pressure, pulse, serum creatinine, Killip classification at admission, cardiac arrest at admission, markers of myocardial necrosis and changes in ST-segment (7). These eight independent risk factors of prognosis are not only a predictive value for risk stratification and nosocomial adverse outcomes in patients with ACS, but also has a significant predictive power in both 
short- and long-term major adverse cardiovascular events (MACE), including all-cause mortality (8). Jakimov et al (9) showed that the GRACE score at admission is an independent predictor of MACE over a 30-day follow-up period. Xiang et al (10) showed that the GRACE score at admission was an independent predictor of long-term MACE in patients with AMI. The GRACE score is a comprehensive assessment system guiding clinical diagnosis, treatment and prognosis evaluation. However, there are some limitations, including a lack of biomarkers that reflect thrombosis and inflammation $(11,12)$.

Previous findings have shown that thrombosis and inflammation play central roles in the occurrence, progression, rupture and thrombosis of atherosclerotic plaques (13). Platelets form an important link between inflammatory reactions and thrombosis (14). At admission, large platelets actively participate in metabolism and enzyme activity compared with smaller platelets, which have greater thrombosis potential (14). The mean platelet volume (MPV) is easy to measure, is a stable parameter of platelet activation and aggregation, and plays an important role in predicting the adverse outcomes in patients with STEMI $(15,16)$. Lymphocytes are one of the earliest cell types involved in atherosclerotic plaque formation and are important biomarkers in determining the inflammatory state of the body (17). Lymphocytes have multiple functions, including producing immunoglobulin $\mathrm{M}$ antibodies, recognizing and oxidizing low-density lipoprotein, and preventing atherosclerosis $(17,18)$. Therefore, in the present study, the mean platelet volume to lymphocyte ratio (MPVLR) was used as a new potential biomarker for inflammation and thrombosis. Recent studies have reported that high MPVLR values at admission are associated with various short-term and long-term adverse outcomes in patients with STEMI after PCI $(19,20)$. However, there are no previous studies evaluating the association between MPVLR and GRACE score, and the combined value of MPVLR with GRACE score in predicting the prognosis of patients with STEMI after PCI. Therefore, aims of the present study were to assess the potential association between MPVLR and GRACE score, and to investigate whether combined MPVLR and GRACE score is a powerful predictor of short-term MACE in patients with STEMI after PCI.

\section{Materials and methods}

Study population. This study was retrospective and conducted at the First Affiliated Hospital of Shihezi University Medical College from October 2017 to January 2019, enrolling 556 patients (including 321 males and 235 females, aged between 20 and 90 years) diagnosed with STEMI who underwent primary PCI within $12 \mathrm{~h}$. The study was approved by The Ethics Committee of The First Affiliated Hospital of Shihezi University School of Medicine.

STEMI was diagnosed based on the American College of Cardiology (21) and included the following criteria: i) Chest pain symptoms occurring within $24 \mathrm{~h}$ prior to admission and lasting for $>30 \mathrm{~min}$; ii) an electrocardiogram showing ST-segment elevation in $\geq 2$ consecutive leads and/or an abnormal Q wave and new left bundle-branch block; and iii) serum biochemical marker creatinine kinase-myocardial band isoenzyme (CK-MB) and/or cardiac troponin T (cTnT) is positively elevated within $24 \mathrm{~h}$ after onset of the symptoms. The following patients were excluded to avoid any factors that could have affected MPVLR: i) Patients with autoimmune diseases $(n=8)$; ii) congenital heart diseases $(n=4)$; iii) cancer $(n=17)$; iv) acute and chronic infectious diseases $(n=13)$; v) severe liver and kidney dysfunction diseases $(n=19)$; vi) those taking steroid drugs within 3 months $(n=10)$ : vii) those who previously underwent PCI $(n=13)$; viii) patients with incomplete clinical data $(n=5)$; ix) medication is not regularly taken; and $\mathrm{x}$ ) poor compliance. Out of 556 patients initially enrolled, 464 patients met the inclusion criteria, and all provided written informed consent. 'Prodromal angina' (PA) was defined as a chest pain episode typically limited to $24 \mathrm{~h}$ before infarction (22). No-reflow was defined as the absence of effective perfusion of myocardial tissue (TIMI flow-grade lower than 3) after coronary artery recanalization without obvious spasm, dissection and residual stenosis (23).

Study procedures and clinical data. Peripheral venous blood samples $(4 \mathrm{ml})$ were collected from patients prior to PCI. Hematological and biochemical analyses were performed using fresh whole blood and plasma within $30 \mathrm{~min}$ of collection. The hematological parameters included testing for the neutrophil count, lymphocyte count, highly sensitive c-reactive protein (hsCRP) and MPV, which were measured using an XT-4000 automated hematology analyzer (Sysmex Corporation). The biochemical indicators analyzed included blood glucose, cTnT, CK-MB and N-terminal pro-brain natriuretic peptide (NT-proBNP). CK-MB, cTnT and NT-proBNP were determined using a Roche E601 immunology analyzer (Roche Diagnostics), while blood glucose was measured using an Hitachi7180 automatic biochemical analyzer (Hitachi, Ltd.). MPVLR was calculated based on the ratio of mean platelet volume to lymphocyte count at admission (20). Using a computer program on the national chest pain center platform (https://datacs.chinacpc.org), the first attending physician recorded the GRACE scores of all the patients at admission. All the patients underwent Philips iE33 transthoracic echocardiography (Philips Healthcare) to assess left ventricular ejection fraction (LVEF) within $24 \mathrm{~h}$ after PCI.

Prior to PCI, all the patients received $300 \mathrm{mg}$ clopidogrel (Sanofi S.A.), $300 \mathrm{mg}$ aspirin (Bayer), and after PCI received daily doses of $75 \mathrm{mg}$ clopidogrel and $100 \mathrm{mg}$ aspirin. The use and dosage of other cardiac drugs were determined by the clinician according to the clinical guidelines formulated by the American College of Cardiology and the condition of the patient (21). The success of PCI was assessed via thrombolysis in myocardial infarction flow grade level 3 after coronary artery therapy and residual stenosis of $<30 \%$ (20). Coronary angiography, PCI and reperfusion therapy strategies were performed by experienced cardiologists.

Primary endpoint and follow-up. Follow up of patients was completed by reviewing hospital records, outpatient visits and telephone contact. The main outcome was that MACE occurred during the follow-up period. MACE included: Cardiogenic or all-cause mortality, malignant arrhythmias (ventricular tachycardia, ventricular fibrillation, grade III atrioventricular block), recurrent myocardial infarction, recurrent angina, 


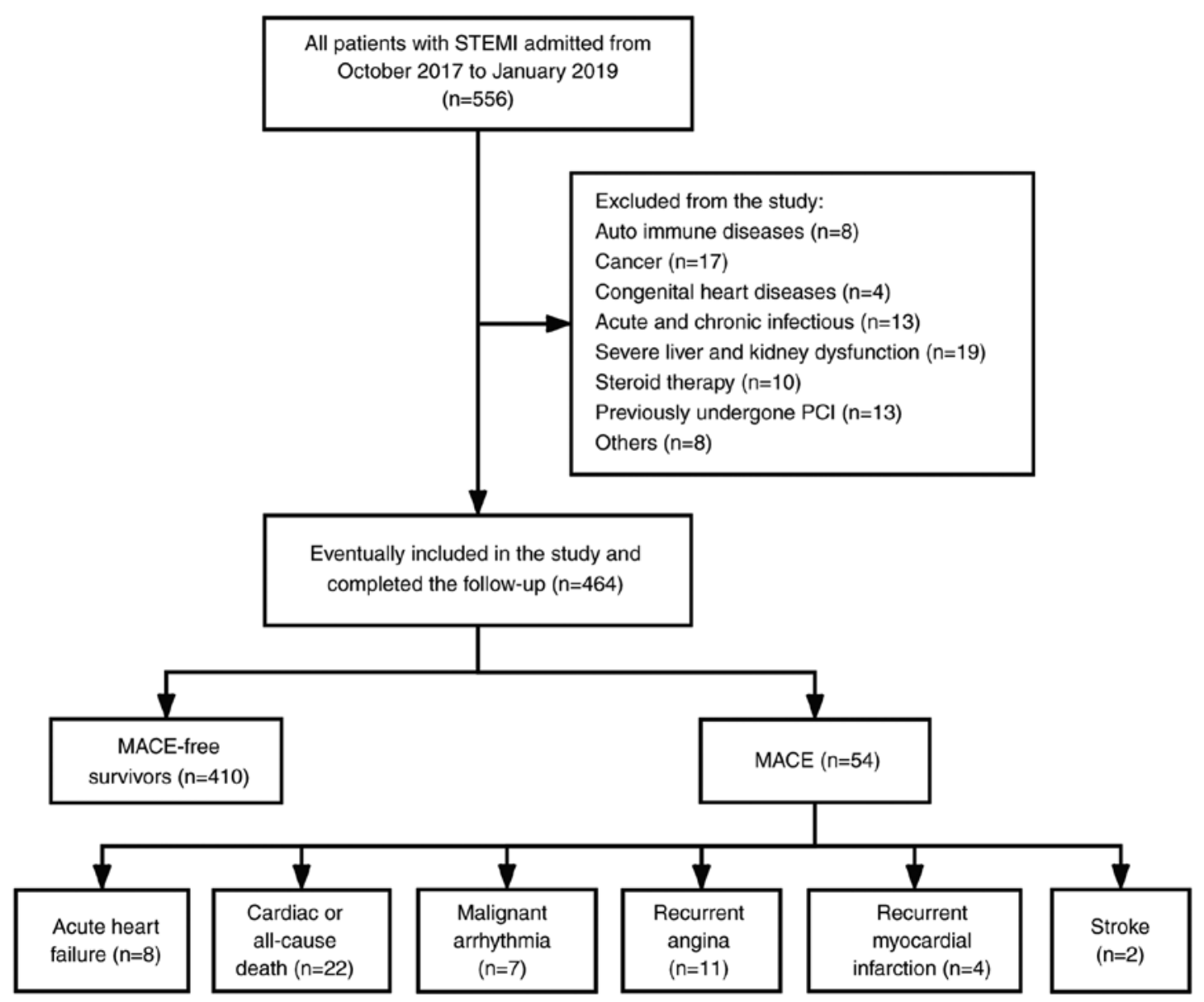

Figure 1. Flow chart of the study cohort. The flow chart presents the selection criteria, exclusion criteria and clinical layout of the study population (MACE group and MACE-free group). MACE, major adverse cardiovascular events.

acute heart failure and stroke (neurological disorders related to recent ischemic or hemorrhagic events) (24).

Statistical analysis. The Kolmogorov-Smirnov test was used to determine the normality of each of the random samples. Mean and standard deviation were used to describe the numerical variables following a normal distribution. However, the median and interquartile range were used to describe numerical variables not following normal distribution. The t-test or Mann-Whitney $\mathrm{U}$ test were used to compare the numerical variables between two groups, while one-way ANOVA was used to compare the numerical variables among multiple groups. When differences between the two groups were needed to be compared using ANOVA, Tukey's post-hoc test was used if equal variances were assumed, and Games-Howell post-hoc test was used if equal variances were not assumed. Non-normally distributed data were analyzed with the Kruskal-Wallis non-parametric test, followed by Dunn's post-hoc test. Frequencies and percentages were used to describe nominal variables, and comparisons between groups performed using $\chi^{2}$ test or Fisher exact probability method. Spearman's rank correlation was utilized to determine the correlation between MPVLR and GRACE scores. A receiver operating characteristic curve (ROC) was used to analyze the value of MPVLR, GRACE score and their combination in predicting MACE and angiographic no-reflow occurrence. Delong's test was used to compare the area under
ROC curve (AUC). Kaplan-Meier analysis method was used to estimate the MACE-free survival rate based on the cut-off values of MPVLR and GRACE scores. The log-rank test was used to compare the MACE free survival rate between groups. Cox regression models were used to evaluate independent risk factors for short-term MACE in patients with STEMI after PCI. Univariate analysis $\mathrm{P}<0.1$ factors were included in multivariate Cox regression analysis. $\mathrm{P}<0.05$ was considered to indicate a statistically significant difference.

\section{Results}

Baseline clinical characteristics. The present study enrolled 464 patients with STEMI after PCI and the median follow-up period was 22 days (range: $1-30$ ). Fig. 1 shows the patient selection flow chart. ROC analyses results indicated that the cut-off values of MPVLR and GRACE score for differentiating short-term MACE were 5.38 (sensitivity $=85.2 \%$; specificity $=64.6 \%$; $\mathrm{P}<0.001$ ) and 145 (sensitivity $=88.9 \%$; specificity $=62.7 \%$; $\mathrm{P}=0.005)$. Based on the optimal cut-off values of MPVLR and GRACE score, patients were segregated into four groups (25): i) Low MPVLR + low GRACE score (Group 1; MPVLR $\leq 5.38$; GRACE score $\leq 145$; $n=181$ ); ii) low MPVLR + high GRACE score (Group 2; MPVLR $\leq 5.38$; GRACE score $>145$; $n=91$ ); iii) high MPVLR + low GRACE score (Group 3; MPVLR >5.38; GRACE score $\leq 145 ; n=93$ ); and iv) high MPVLR + high 
Table I. Comparison of baseline clinical characteristics of patients divided into groups based on the different MPVLR combined with GRACE scores.

\begin{tabular}{|c|c|c|c|c|c|}
\hline Variable & $\begin{array}{l}\text { Low MPVLR + } \\
\text { low GRACE } \\
\text { score (Group 1, } \\
\text { n=181) }\end{array}$ & $\begin{array}{c}\text { Low MPVLR + } \\
\text { high GRACE } \\
\text { score (Group 2, } \\
\text { n=91) }\end{array}$ & $\begin{array}{c}\text { Low MPVLR + } \\
\text { high GRACE } \\
\text { score (Group 3, } \\
\text { n=93) }\end{array}$ & $\begin{array}{c}\text { High MPVLR + } \\
\text { high GRACE } \\
\text { score (Group 4, } \\
\text { n=99) }\end{array}$ & P-value \\
\hline \multicolumn{6}{|l|}{ Baseline characteristics } \\
\hline Age, years & $53.68 \pm 7.91$ & $55.43 \pm 8.26$ & $53.06 \pm 8.94$ & $59.42 \pm 9.99^{\mathrm{a}-\mathrm{c}}$ & $<0.001$ \\
\hline Male, n (\%) & $105(58.01)$ & $55(60.44)$ & $51(54.84)$ & $57(57.58)$ & 0.897 \\
\hline Current smokers, n (\%) & $94(51.93)$ & $52(57.14)$ & $41(44.09)$ & $53(53.54)$ & 0.337 \\
\hline Hypertension, n (\%) & $121(66.85)$ & $63(69.23)$ & $65(69.89)$ & $81(81.82)$ & 0.062 \\
\hline Diabetes mellitus, n (\%) & 49 (27.07) & $27(29.67)$ & $35(37.63)^{\mathrm{a}, \mathrm{b}}$ & $45(45.45)^{\mathrm{a}-\mathrm{c}}$ & 0.012 \\
\hline Dyslipidaemia, n (\%) & $52(28.73)$ & $15(16.48)$ & $23(24.73)$ & $32(32.32)$ & 0.179 \\
\hline Prodromal angina, $\mathrm{n}(\%)$ & $60(33.15)$ & $28(30.77)$ & $30(32.26)$ & $17\left(17.17^{\mathrm{a}-\mathrm{c}}\right.$ & 0.032 \\
\hline Killip class $\geq \mathrm{II}, \mathrm{n}(\%)$ & $66(36.46)$ & $35(38.46)$ & $40(43.01)^{\mathrm{a}, \mathrm{b}}$ & $55(55.56)^{\mathrm{a}-\mathrm{c}}$ & 0.017 \\
\hline GRACE score & $129.52 \pm 13.95$ & $156.82 \pm 12.70^{\mathrm{a}}$ & $120.32 \pm 17.32^{\mathrm{a}, \mathrm{b}}$ & $167.24 \pm 14.86^{\mathrm{a}-\mathrm{c}}$ & 0.001 \\
\hline \multicolumn{6}{|l|}{ Laboratory data } \\
\hline Neutrophil count, $\mathrm{x} 10^{9} / 1$ & $6.03 \pm 2.68$ & $6.21 \pm 3.42$ & $6.44 \pm 2.88^{\mathrm{a}}$ & $7.43 \pm 3.75^{\mathrm{a}-\mathrm{c}}$ & 0.004 \\
\hline Lymphocyte count, $\mathrm{x} 10^{9} / 1$ & $2.84 \pm 0.64$ & $2.81 \pm 0.77$ & $1.59 \pm 0.30^{\mathrm{a}, \mathrm{b}}$ & $1.57 \pm 0.37^{\mathrm{a}, \mathrm{b}}$ & $<0.001$ \\
\hline NLR & $2.25 \pm 1.20$ & $2.44 \pm 1.70$ & $4.24 \pm 2.10^{\mathrm{a}, \mathrm{b}}$ & $5.09 \pm 3.05^{\mathrm{a}-\mathrm{c}}$ & $<0.001$ \\
\hline MPV, fl & $10.45 \pm 0.97$ & $10.85 \pm 0.73^{\mathrm{a}}$ & $11.02 \pm 0.93^{\mathrm{a}}$ & $11.00 \pm 1.10^{\mathrm{a}}$ & $<0.001$ \\
\hline MPVLR & $3.83 \pm 0.81$ & $4.08 \pm 0.90$ & $7.21 \pm 1.66^{\mathrm{a}, \mathrm{b}}$ & $7.83 \pm 2.95^{\mathrm{a}-\mathrm{c}}$ & $<0.001$ \\
\hline Peak CK-MB, U/1 & $146(84-249)$ & $86(64-186)$ & $144(66-233)$ & $162(53-375)$ & 0.113 \\
\hline Peak cTnT, ng/ml & $3.64(2.25-5.70)$ & $4.29(2.31-5.89)^{\mathrm{a}}$ & $4.20(2.82-6.09)^{\mathrm{a}}$ & $6.85(2.72-8.99)^{a-c}$ & $<0.001$ \\
\hline NT-proBNP, pg/ml & $885(494-2,499)$ & $1,366(741-3,215)^{\mathrm{a}}$ & $1,239(764-2,519)^{\mathrm{a}}$ & $3,610(1,750-6,800)^{a-c}$ & $<0.001$ \\
\hline Glu, mmol/1 & $7.32 \pm 3.93$ & $7.73 \pm 3.54$ & $7.97 \pm 4.40$ & $7.92 \pm 3.22$ & 0.476 \\
\hline hsCRP, mg/l & $2.60(1.40-9.38)$ & $1.40(0.87-3.20)^{\mathrm{a}}$ & $1.72(0.85-5.50)^{\mathrm{a}}$ & $2.70(1.64-5.45)^{\mathrm{b}, \mathrm{c}}$ & 0.002 \\
\hline LVEF & $59.52 \pm 9.24$ & $59.46 \pm 10.81$ & $57.56 \pm 8.53^{\mathrm{a}, \mathrm{b}}$ & $52.65 \pm 10.19^{\mathrm{a}-\mathrm{c}}$ & $<0.001$ \\
\hline Culprit vessel, n (\%) & & & & & 0.327 \\
\hline Right coronary artery & $80(44.20)$ & $30(32.97)$ & $38(40.86)$ & $37(37.37)$ & \\
\hline Left circumflex artery & $23(12.71)$ & $10(10.98)$ & $12(12.90)$ & $13(13.13)$ & \\
\hline Left anterior descending artery & $78(43.09)$ & $49(53.58)$ & $43(46.24)$ & $47(47.47)$ & \\
\hline Left main coronary artery & $0(0)$ & $2(2.20)$ & $0(0)$ & $2(2.03)$ & \\
\hline Number of implanted stents, $n$ & $1.21 \pm 0.57$ & $1.31 \pm 0.59$ & $1.17 \pm 0.50$ & $1.22 \pm 0.56$ & 0.942 \\
\hline \multicolumn{6}{|l|}{ Postoperative medication, n (\%) } \\
\hline Clopidogrel & $167(92.23)$ & $86(94.51)$ & $88(94.62)$ & $92(92.93)$ & 0.848 \\
\hline Aspirin & $173(95.58)$ & $85(93.41)$ & $89(95.70)$ & $96(96.97)$ & 0.709 \\
\hline Statin & $153(84.53)$ & $79(86.81)$ & $78(83.87)$ & $90(90.91)$ & 0.436 \\
\hline Beta-blocker & $144(79.56)$ & $68(74.73)$ & $73(78.49)$ & $80(80.81)$ & 0.753 \\
\hline ACEI or ARB & $90(49.72)$ & $43(47.25)$ & $49(52.69)$ & $55(55.56)$ & 0.669 \\
\hline Calcium channel blocker & $40(22.10)$ & $18(19.78)$ & $22(23.68)$ & $30(30.30)$ & 0.332 \\
\hline
\end{tabular}

MPVLR, mean platelet volume to lymphocyte ratio; GRACE, global registry of acute coronary events; NLR, neutrophil to lymphocyte ratio; MPV, mean platelet volume; CK-MB, creatine kinase isozyme; cTnT, cardia troponin T; NT-proBNP, n-terminal brain natriuretic peptide precursor; Glu, fasting blood sugar; hsCRP, high sensitivity c-reactive protein; LVEF, left ventricular ejection fraction; ACEI, angiotensin-converting enzyme inhibitors; ARB, angiotensin type II receptor blockers. ${ }^{a} \mathrm{P}<0.05$ vs. low MPVLR + low GRACE. ${ }^{\text {b }}<0.05$ vs. low MPVLR + high GRACE. ${ }^{c} \mathrm{P}<0.05$ vs. high MPVLR + low GRACE.

GRACE score (Group 4; MPVLR >5.38; GRACE score >145; $\mathrm{n}=99)$. The basic clinical and procedural characteristics of the four groups of patients are shown in Table I. Patients in Group 4 were significantly older, had a higher admission GRACE score, Killip class, neutrophil count, neutrophil to lymphocyte ratio
(NLR), MPV, MPVLR, peak of cTnT, NT-proBNP and hsCRP, and lower levels of LVEF and lymphocyte count compared to patients in Group 1. Patients in Group 4 also had a higher proportion of diabetes mellitus and PA compared with the other three groups. Moreover, there were no significant differences in 
Table II. Comparison of baseline clinical characteristics of patients in the MACE and MACE-free groups.

\begin{tabular}{|c|c|c|c|}
\hline Variable & MACE group $(n=54)$ & MACE-free group $(n=410)$ & P-value \\
\hline \multicolumn{4}{|l|}{ Baseline characteristics } \\
\hline Age, years & $71.19 \pm 11.29$ & $58.11 \pm 10.69$ & $<0.001$ \\
\hline Male, n (\%) & $32(59.26)$ & $236(57.56)$ & 0.812 \\
\hline Current smokers, n (\%) & $34(62.96)$ & $206(50.24)$ & 0.079 \\
\hline Hypertension, $\mathrm{n}(\%)$ & $44(81.48)$ & $286(69.76)$ & 0.074 \\
\hline Diabetes mellitus, $\mathrm{n}(\%)$ & $28(51.85)$ & $128(31.22)$ & 0.003 \\
\hline Dyslipidaemia, n (\%) & $19(35.19)$ & $103(25.12)$ & 0.114 \\
\hline Prodromal angina, n (\%) & $11(20.37)$ & $144(35.12)$ & 0.031 \\
\hline Killip class $\geq \mathrm{II}, \mathrm{n}(\%)$ & $30(55.56)$ & $166(40.49)$ & 0.035 \\
\hline GRACE score & $165.31 \pm 17.32$ & $138.56 \pm 24.06$ & $<0.001$ \\
\hline \multicolumn{4}{|l|}{ Laboratory data } \\
\hline Neutrophil count, $\times 10^{9} / 1$ & $8.19 \pm 3.17$ & $6.49 \pm 3.18$ & $<0.001$ \\
\hline Lymphocyte count, $\times 10^{9} / 1$ & $1.70(1.38-2.00)$ & $2.30(1.80-2.90)$ & $<0.001$ \\
\hline NLR & $4.61(3.47-6.40)$ & $2.44(1.53-4.33)$ & $<0.001$ \\
\hline MPV, fl & $10.90(10.60-11.80)$ & $10.70(10.10-11.40)$ & 0.002 \\
\hline MPVLR & $6.39(5.64-8.10)$ & $4.61(3.66-6.00)$ & $<0.001$ \\
\hline Peak CK-MB, U/1 & $166(41-300)$ & $137(69-245)$ & 0.914 \\
\hline Peak cTnT, ng/ml & $7.15(3.67-9.70)$ & $4.09(2.47-6.02)$ & $<0.001$ \\
\hline NT-proBNP, pg/ml & $3,800(1,736-4,602)$ & $1,209(650-2,609)$ & $<0.001$ \\
\hline Glu, $\mathrm{mmol} / \mathrm{l}$ & $8.35 \pm 3.72$ & $7.59 \pm 3.83$ & 0.171 \\
\hline hsCRP, $\mathrm{mg} / \mathrm{l}$ & $3.25(1.58-8.28)$ & $2.25(1.10-5.71)$ & 0.041 \\
\hline LVEF & $48.94 \pm 8.46$ & $58.79 \pm 9.61$ & $<0.001$ \\
\hline \multicolumn{4}{|l|}{ Culprit vessel, n (\%) } \\
\hline Right coronary artery & $18(33.33)$ & $160(39.02)$ & 0.419 \\
\hline Left circumflex artery & $8(14.81)$ & $67(16.34)$ & 0.775 \\
\hline Left anterior descending artery & $27(50.00)$ & $180(43.90)$ & 0.397 \\
\hline Left main coronary artery & $1(1.85)$ & $3(0.73)$ & 0.403 \\
\hline Number of implanted stents, $\mathrm{n}$ & $1.25 \pm 0.65$ & $1.22 \pm 0.55$ & 0.770 \\
\hline \multicolumn{4}{|l|}{ Postoperative medication, n (\%) } \\
\hline Clopidogrel & $50(92.59)$ & $383(93.41)$ & 0.820 \\
\hline Aspirin & $51(94.44)$ & $392(95.61)$ & 0.699 \\
\hline Statin & $46(85.19)$ & $354(86.34)$ & 0.817 \\
\hline Beta-blocker & $42(77.78)$ & $323(78.78)$ & 0.866 \\
\hline ACEI or ARB & $26(48.15)$ & $211(51.46)$ & 0.647 \\
\hline Calcium channel blocker & $12(22.22)$ & $98(23.90)$ & 0.785 \\
\hline
\end{tabular}

MACE, major adverse cardiovascular events; MPVLR, mean platelet volume to lymphocyte ratio; GRACE, global registry of acute coronary events; NLR, neutrophil to lymphocyte ratio; MPV, mean platelet volume; CK-MB, creatine kinase isozyme; cTnT, cardia troponin T; NT-proBNP, n-terminal brain natriuretic peptide precursor; Glu, fasting blood sugar; hsCRP, high sensitivity c-reactive protein; LVEF, left ventricular ejection fraction; ACEI, angiotensin-converting enzyme inhibitors; ARB, angiotensin type II receptor blockers.

coronary angiography and postoperative medication among the four groups.

Comparison of baseline clinical characteristics of patients in the MACE and MACE-free groups. Table II shows the baseline clinical characteristics of patients in the MACE and MACE-free groups. Compared to the MACE-free group, the MACE group patients were older, had higher neutrophil count, NLR, MPV, peak of cTnT, NT-proBNP, hsCRP, and also a higher proportion of diabetes and killip class $\geq$ II. However, patients with MACE had a lower proportion of PA, lymphocyte count and LVEF. Moreover, the MPVLR and GRACE score of patients in the MACE group were significantly higher compared with the MACE-free group.

Clinical adverse outcomes. During the follow-up period, 54 (11.64\%) patients experienced MACE. These included 22 (4.74\%) cardiac or all-cause mortality, seven (1.51\%) malignant arrhythmia, $11(2.37 \%)$ recurrent angina, four $(0.86 \%)$ recurrent myocardial infarction, eight $(1.72 \%)$ acute heart failure 
Table III. Comparison of adverse outcomes among the four groups based on the MPVLR and GRACE score cut-off.

\begin{tabular}{lccccr}
\hline Variable & $\begin{array}{c}\text { Low MPVLR } \\
\text { ow GRACE } \\
\text { score (Group 1, } \\
\mathrm{n}=181)\end{array}$ & $\begin{array}{c}\text { Low MPVLR+ } \\
\text { high GRACE } \\
\text { score (Group 2, } \\
\mathrm{n=91)}\end{array}$ & $\begin{array}{c}\text { High MPVLR + } \\
\text { low GRACE } \\
\text { score (Group 3, } \\
\mathrm{n=93)}\end{array}$ & $\begin{array}{c}\text { High MPVLR + } \\
\text { high GRACE } \\
\text { score (Group 4, } \\
\text { n=99) }\end{array}$ & P-value \\
\hline Angiographic no-reflow, n (\%) & $8(4.42)$ & $13(14.29)^{\mathrm{a}}$ & $12(12.90)^{\mathrm{a}}$ & $26(26.26)^{\mathrm{a}-\mathrm{c}}$ & $<0.001$ \\
MACE during follow-up, n (\%) & $6(3.30)$ & $10(11.00)^{\mathrm{a}}$ & $13(13.98)^{\mathrm{a}}$ & $25(25.25)^{\mathrm{a}-\mathrm{c}}$ & $<0.001$ \\
Cardiac or all-cause death & $2(1.10)$ & $3(3.30)$ & $4(4.29)$ & $13(13.13)^{\mathrm{a}-\mathrm{c}}$ & $<0.001$ \\
Malignant arrhythmia & $1(0.55)$ & $2(2.20)$ & $1(1.08)$ & $3(3.03)$ & 0.307 \\
Recurrent angina & $0(0.00)$ & $3(3.30)$ & $2(2.15)$ & $6(6.07)^{\mathrm{a}-\mathrm{c}}$ & 0.003 \\
Recurrent myocardial infarction & $1(0.55)$ & $0(0.00)$ & $2(2.15)$ & $1(1.01)$ & 0.424 \\
Acute heart failure & $2(1.10)$ & $2(2.20)$ & $3(3.23)$ & $1(1.01)$ & 0.552 \\
Stroke & $0(0.00)$ & $0(0.00)$ & $1(1.08)$ & $1(1.01)$ & 0.366 \\
\hline
\end{tabular}

MACE, major adverse cardiovascular events; MPVLR, mean platelet volume to lymphocyte ratio; GRACE, global registry of acute coronary events. ${ }^{a} \mathrm{P}<0.05$ vs. low MPVLR + low GRACE. ${ }^{\mathrm{b}} \mathrm{P}<0.05$ vs. low MPVLR + high GRACE. ${ }^{\mathrm{c}} \mathrm{P}<0.05$ vs. high MPVLR + low GRACE.
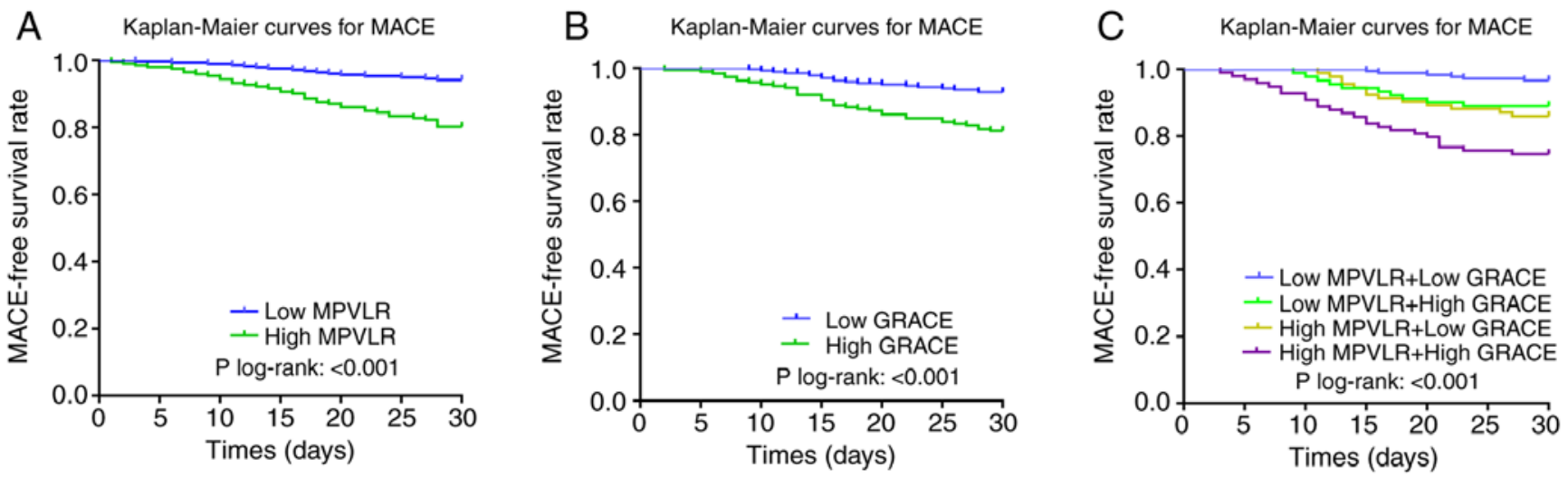

Figure 2. Kaplan-Meier survival curves of 30-day MACE in patients with ST-segment elevation myocardial infarction after percutaneous coronary intervention according to the cut-off value of (A) MPVLR and (B) GRACE scores, and (C) according to the combination of MPVLR and GRACE scores. MACE, major adverse cardiovascular events; MPVLR, mean platelet volume to lymphocyte ratio; GRACE, global registry of acute coronary events.

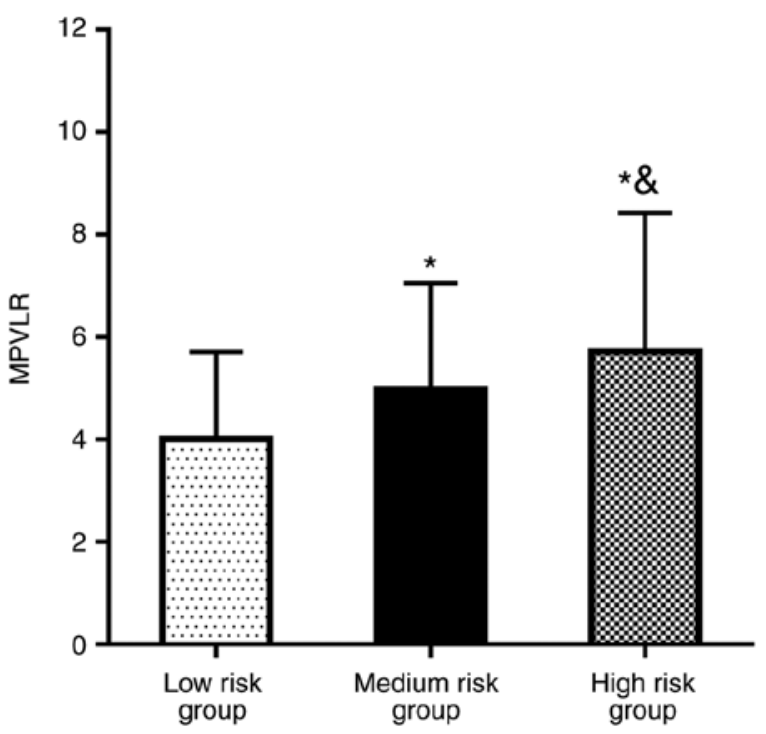

Figure 3. Comparison of MPVLR levels in each risk stratification of global registry of acute coronary events score. MPVLR, mean platelet volume to lymphocyte ratio. ${ }^{*} \mathrm{P}<0.05$ vs. low risk group; ${ }^{\circledR} \mathrm{P}<0.05$ vs. medium risk group.

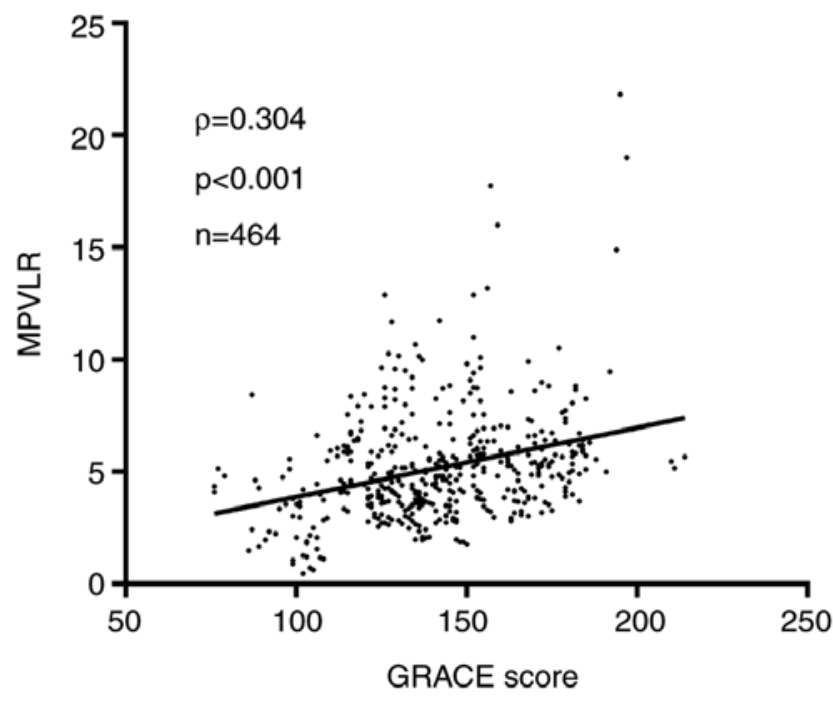

Figure 4. A scatter plot showing the correlation between MPVLR and GRACE score. @, spearman's rank correlation coefficient. MPVLR, mean platelet volume to lymphocyte ratio; GRACE, global registry of acute coronary events. 
Table IV. Cox regression analysis of risk factors for MACE in patients during follow-up.

\begin{tabular}{|c|c|c|c|c|c|c|}
\hline \multirow[b]{3}{*}{ Variable } & \multirow{2}{*}{\multicolumn{2}{|c|}{ Univariate analysis }} & \multicolumn{4}{|c|}{ Multivariate analysis } \\
\hline & & & \multicolumn{2}{|l|}{ Model 1} & \multicolumn{2}{|l|}{ Model 2} \\
\hline & $\mathrm{HR}(95 \% \mathrm{CI})$ & P-value & $\mathrm{HR}(95 \% \mathrm{CI})$ & P-value & $\mathrm{HR}(95 \% \mathrm{CI})$ & P-value \\
\hline Age & $1.088(1.062-1.234)$ & $<0.001$ & $1.055(1.025-1.131)$ & $<0.001$ & $1.048(1.017-1.113)$ & 0.002 \\
\hline Diabetes mellitus & $1.453(0.921-1.964)$ & 0.236 & - & - & - & - \\
\hline Prodromal angina & $0.946(0.795-0.991)$ & 0.042 & $0.910(0.839-0.963)$ & 0.026 & $0.885(0.816-0.947)$ & 0.021 \\
\hline Killip class $\geq$ II & $1.786(1.036-2.886)$ & 0.036 & $1.721(1.324-2.320)$ & 0.019 & $1.611(1.266-1.897)$ & 0.026 \\
\hline NLR & $1.315(1.144-1.545)$ & $<0.001$ & $1.152(1.089-1.418)$ & 0.020 & $1.128(1.102-1.407)$ & 0.012 \\
\hline hsCRP & $1.485(1.122-1.676)$ & 0.042 & $1.233(1.076-1.538)$ & 0.033 & $1.184(1.076-1.510)$ & 0.019 \\
\hline NT-proBNP & $1.032(1.001-1.095)$ & $<0.001$ & $1.008(1.000-1.039)$ & 0.027 & $1.005(1.002-1.027)$ & 0.013 \\
\hline Peak cTnT & $1.471(1.086-1.787)$ & $<0.001$ & $1.325(0.987-1.880)$ & 0.521 & $1.252(0.932-1.780)$ & 0.475 \\
\hline LVEF & $0.928(0.863-0.953)$ & $<0.001$ & $0.911(0.895-0.947)$ & $<0.001$ & $0.903(0.880-0.928)$ & $<0.001$ \\
\hline MPVLR $>5.38$ & $2.987(2.247-4.568)$ & $<0.001$ & $1.668(1.202-2.170)$ & $<0.001$ & - & - \\
\hline GRACE $>145$ & $3.102(1.691-4.926)$ & $<0.001$ & $1.706(1.435-3.058)$ & $<0.001$ & - & - \\
\hline Low MPVLR+ high GRACE & $2.105(1.358-6.281)$ & 0.013 & - & - & $1.625(1.168-2.609)$ & 0.007 \\
\hline High MPVLR + low GRACE & $2.558(1.637-7.002)$ & 0.022 & - & - & $1.806(1.392-2.809)$ & 0.018 \\
\hline High MPVLR + high GRACE & $5.382(3.745-8.753)$ & $<0.001$ & - & - & $2.455(1.736-3.188)$ & $<0.001$ \\
\hline
\end{tabular}

MPVLR, mean platelet volume to lymphocyte ratio; GRACE, global registry of acute coronary events; NLR, neutrophil to lymphocyte ratio; hsCRP, high sensitivity c-reactive protein; cTnT, cardiac troponin T; NT-proBNP, n-terminal brain natriuretic peptide precursor; LVEF, left ventricular ejection fraction; HR, hazard ratio.

and two $(0.43 \%)$ strokes. The present results suggested that the incidence of MACE and angiographic no-reflow during follow-up were significantly increased in the high MPVLR + high GRACE score group compared with the other three groups. In terms of cardiac or all-cause mortality and recurrent angina, these were significantly increased in patients in the high MPVLR + high GRACE score group compared with the other three groups. However, malignant arrhythmia, recurrent angina, acute heart failure and stroke were similar in the four groups (Table III).

Kaplan-Meier survival curves based on the cut-off values of MPVLR and GRACE score are shown in Fig. 2A and B, respectively. The rate of MACE in the high MPVLR group (19.79 vs. 5.88\%; log-rank, $\mathrm{P}<0.001$; Fig. $2 \mathrm{~A}$ ) and the high GRACE score group (18.42 vs. $6.93 \%$; log-rank, $\mathrm{P}<0.001$; Fig. 2B) increased significantly compared with the control group during the follow-up period. In addition, Kaplan-Meier survival curves based on the combined biomarkers (MPVLR and GR ACE scores) are shown in Fig. 2C. There was a significant intergroup difference in short-term MACE among the four groups, and the short-term MACE in the high MPVLR+ high GRACE score group was increased compared with the other three groups (high MPVLR + high GRACE score vs. high MPVLR + low GRACE score vs. low MPVLR + high GRACE score; 25.25 vs. 13.98 vs. $11.00 \%$, respectively; $\mathrm{P}<0.001$; Fig. 2C).

Independent predictors factors for short-term MACE. Cox proportional hazard analysis was used to construct model 1 and model 2 for prediction of the risk factors for short-term MACE after PCI in patients with STEMI (Table IV). Univariate analysis results suggested that age, prodromal angina, Killip class $\geq I I$, NLR, hsCRP, NT-proBNP, the peak of cTnT, LVEF and combined MPVLR with GRACE score were all associated with a 30-day MACE. After adjusting the covariates in model 1, high GRACE score (HR, 1.706; 95\% CI, 1.435-3.058; $\mathrm{P}<0.001)$ and high MPVLR (HR, 1.668; 95\% CI, 1.202-2.170; $\mathrm{P}<0.001)$ were significant independent predictors of a 30-day MACE. Multivariate Cox analysis in model 2 showed that the combination of high GRACE score with high MPVLR (HR, 2.455 ; 95\% CI, 1.736-3.188; $\mathrm{P}<0.001$ ) was a powerful predictor of a 30-day MACE.

Correlation between MPVLR and GRACE score. Based on the GRACE score, patients were categorized into three groups: High-risk group (GRACE score $>140 ; n=213$ ), medium-risk group (108< GRACE score $\leq 140 ; n=161)$ and low-risk group (GRACE score $\leq 108 ; n=90$ ). It was demonstrated that, with increased GRACE risk stratification, the MPVLR level of each group increased significantly $(\mathrm{P}<0.05$; Fig. 3$)$. In addition, Spearman's rank correlation results indicated that there was a significant linear correlation between MPVLR with GRACE score ( $\mathrm{Q}=0.304 ; \mathrm{P}<0.001 ;$ Fig. 4).

Combination of MPVLR with GRACE score in predicting clinical adverse outcomes. ROC curves assessed and compared the predictive efficacy of MPVLR, GRACE score and their combination in predicting adverse clinical outcomes after PCI in patients with STEMI. As presented in Fig. 5A, a combination of GRACE score with MPVLR (AUC, 0.853 ; 95\% CI, 0.800-0.907) had improved predictive efficacy for short-term MACE, compared with individual MPVLR (AUC, 0.787; 
A

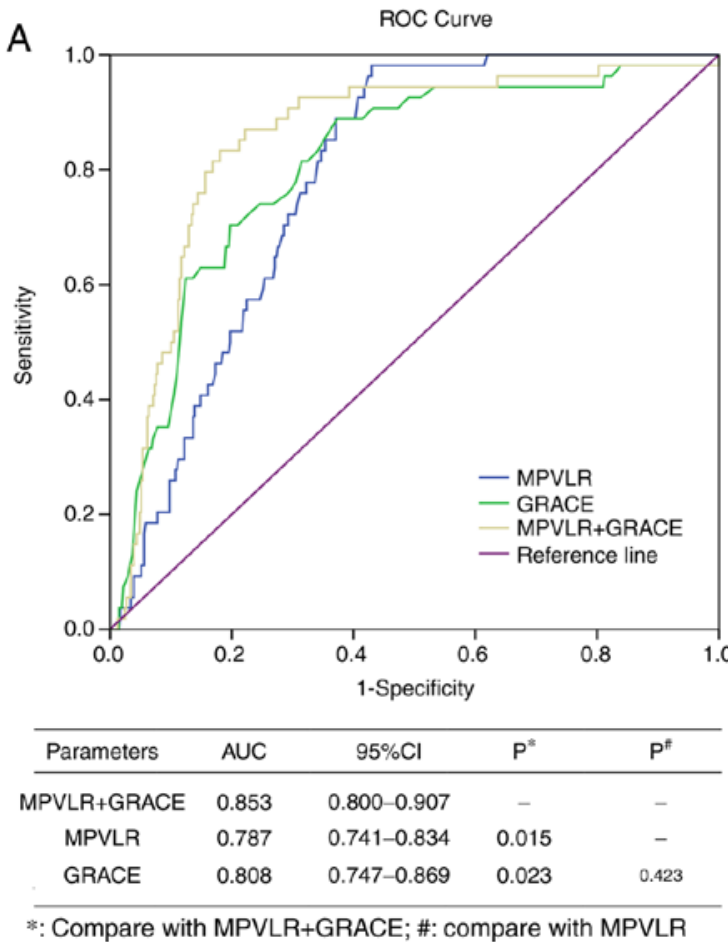

B

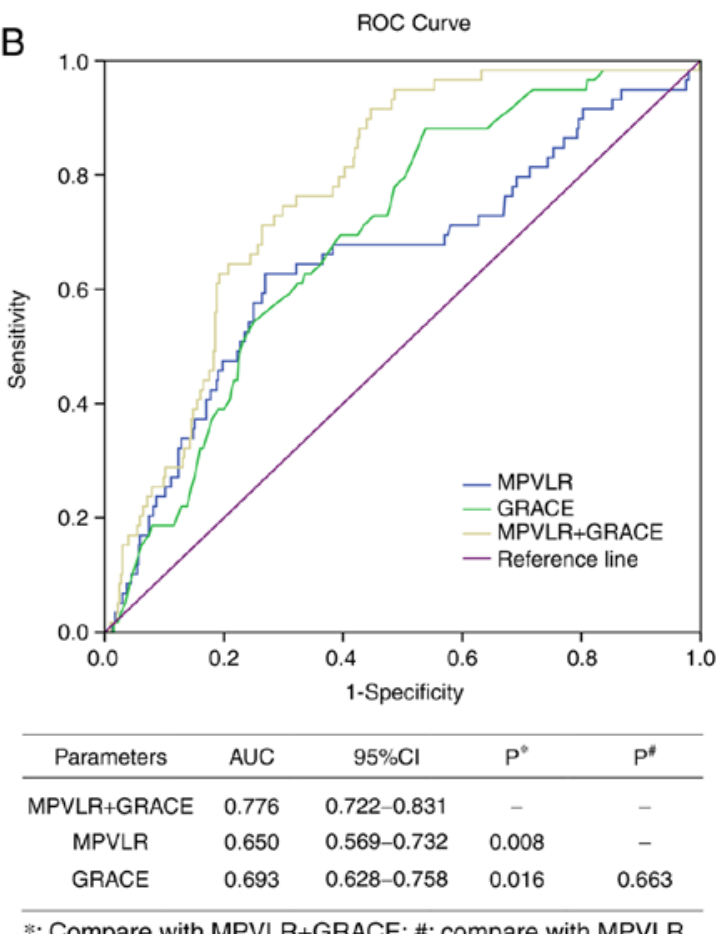

Figure 5. Receiver operating characteristic curves presenting AUC values for MPVLR in combination with GRACE score (MPVLR + GRACE), MPVLR alone and GRACE alone. (A) Short-term MACE and (B) angiographic no-reflow were predicted in patients with STEMI. GRACE, global registry of acute coronary events; MPVLR, mean platelet volume to lymphocyte ratio; MACE, major adverse cardiovascular events; AUC, area under the curve.

95\% CI, 0.741-0.834) and GRACE score (AUC, 0.808; 95\% CI, 0.747-0.869; $\mathrm{P}<0.05)$. In addition, GRACE score together with MPVLR (AUC, 0.776; 95\% CI, 0.722-0.831) significantly improved the prediction efficiency of angiographic no-reflow (Fig. 5B; $\mathrm{P}<0.05$ ) compared with single prediction with MPVLR (AUC, 0.650; 95\% CI, 0.569-0.732) and GRACE score (AUC, 0.693; 95\% CI, 0.628-0.758). Collectively, the present results suggested that the combination of MPVLR and GRACE score may improve the prediction of clinical adverse outcomes in patients with STEMI after PCI.

\section{Discussion}

The present study, not only investigated the potential association between GRACE score and MPVLR, but also compared the predictive value of GRACE score, MPVLR and GRACE score combined with MPVLR for no-reflow and short-term MACE in patients with STEMI after PCI. In addition, the present study also examined the potential mechanism between the loss of PA and the increase in MPVLR and GRACE scores. The present results suggested that MPVLR is a simple, non-invasive, economical and feasible biomarker, which can account for the deficiency of the GRACE score system. It was also indicated that MPVLR has a practical clinical value in predicting the prognosis of patients with STEMI. The present findings demonstrated that MPVLR combined with GRACE score has a more powerful predictive potential for short-term adverse outcomes in patients with STEMI after PCI, compared with an individual MPLVR or GRACE score.

MPV is an important indicator of platelet activation and aggregation (26). Large platelets with more active metabolism can accelerate the formation of coronary thrombosis, and play a considerable role in the pathological and physiological process of AMI (27-29). Previous clinical studies have shown that higher MPV at the time of admission is associated with all-cause mortality and MACE incidence in patients with STEMI (30,31). Goncalves et al (32) showed that patients with ACS have larger MPV and larger platelet metabolism and enzyme activities were higher, which increased the occurrence of adverse outcomes via the release of inflammatory mediators, increased thrombosis, aggravated microvascular dysfunction, inflammation and myocardial injury, microcirculation insufficiency, large infarction area and deterioration of cardiac function. Moreover, Núñez et al found that lymphocytes are involved in the growth, development, rupture and thrombosis of atherosclerotic plaques (33). The decrease in lymphocyte count is related to the body's physiological stress, increased inflammatory response and increased apoptosis (33). In addition, lymphocyte count was found to be important biomarkers of inflammatory reactions in patients with STEMI, and is associated with MACE and angiographic no-reflow (34). The present results suggested that patients in the MACE group had a lower lymphocyte count compared with the MACE-free group. Moreover, patients in the high MPVLR and high GRACE score group showed significantly lower rates of prodromal angina compared with the other three groups. These results are consistent with previous results from Gok et al (35). However, the relationship between PA absence and increases in MPVLR and GRACE scores is not fully understood. PA may be a process of ischemic preconditioning, which can delay the death of myocardial cells and has a protective effect on the myocardia ischemic injured before reperfusion $(22,36)$. PA not only reduces myocardial infarct size but also protects 
microcirculation after reperfusion $(35,37)$. Therefore, the absence of PA in patients with STEMI often indicates that the disease is more serious, and the corresponding GRACE score is higher and with poorer prognosis (38). In addition, inflammation plays an important role in myocardial ischemia-reperfusion injury (38). Therefore, the reason for the higher MPVLR in patients with STEMI in the absence of PA may be associated with the anti-inflammatory effect and inhibition of platelet activation of PA (39).

MPVLR is the ratio of MPV to lymphocyte count, and an increase in MPV and/or a decrease in lymphocyte count can result in increased MPVLR (20). MPVLR is a comprehensive biomarker for thrombosis and inflammation (29). MPVLR combines the advantages of MPV and lymphocytes in indicating the physiological stress in STEMI patients, overcome the shortcomings of each one of them (20). In addition, MPVLR functions as an indicator of the body's inflammatory response and the degree of thrombosis (19). Ornek and Kurtul (40) showed that MPVLR predicted impaired coronary collateral circulation in patients with stable coronary artery disease. Moreover, Kilic and Kurtul (41) showed that elevated MPVLR is associated with the complexity and severity of coronary atherosclerosis in patients with ACS. The present results indicated that MPVLR levels were positively correlated with GRACE score and that patients in the high MPVLR group had a higher incidence of MACE. Therefore, MPVLR may be used as an auxiliary indicator to predict adverse outcome in patients with STEMI.

The GRACE score is the largest and best-known database prospective study used for ACS (42). Patients were enrolled from 30 countries across North and South America, Australia, New Zealand, Asia and Europe, and it has been widely used to identify high-risk patients with AMI and assess prognosis (43). The GRACE score system, however, has some limitations, such as it does not consider the thrombotic activity and inflammatory status of the body (44). Hence, there is a lack of biomarkers related to adverse outcomes. Thus, there is a need for objective biomarkers for the comprehensive evaluation of prognosis in AMI patients. Previous studies have identified several biomarkers outside the scoring system, such as NT-proBNP (45), hsCRP (46), neutrophil count (44), homocysteine and the fibrinogen-albumin ratio $(47,48)$; these have significantly improved the predictive efficacy of GRACE risk score system for adverse outcomes in patients with ACS. The present study investigated the association between MPVLR and GRACE score, and found that MPVLR combined with GRACE score may be used as a powerful and stable predictor for short-term MACE prediction after PCI in patients with STEMI.

The present study had some limitations. First, the present study was a single-center retrospective study with limited sample size and possible selection bias. Moreover, the prognostic value of other biomarkers for patients with STEMI were not investigated. In addition, the changes in MPVLR were not dynamically observed and there was no assessment of whether a similar predictive value was present in MPVLR after treatment.

In conclusion, MPVLR and GRACE score combination on admission showed significant predictive value for short-term MACE after PCI in patients with STEMI. Therefore, this combination may be used to identify high-risk patients with poor prognosis and aid treatment in the early disease stage. The combination of MPVLR, which is a non-invasive, simple, economical and feasible biomarker, and GRACE score provides a new perspective for the assessment, treatment and prognosis of patients with STEMI.

\section{Acknowledgements}

The authors would like to thank Professor Guihua Li, Director of The Department of Emergency (First Affiliated Hospital, Shihezi University School of Medicine) for providing valuable suggestions for the present study.

\section{Funding}

No funding was received.

\section{Availability of data and materials}

The datasets used and/or analyzed during the current study are available from the corresponding author on reasonable request.

\section{Authors' contributions}

XC, MS and GL contributed to the conception and design of the study, and drafted the manuscript. TZ and YM contributed to the collection, collation and statistical analysis of data. WZ, $\mathrm{HZ}$ and $\mathrm{HH}$ contributed to the feasibility analysis of the study. $\mathrm{XC}$ and GL revised the manuscript. GL is responsible for study supervision and management.

\section{Ethics approval and consent to participate}

The present study was approved by The Ethics Committee of The First Affiliated Hospital of Shihezi University School of Medicine. All patients provided written informed consent.

\section{Patient consent for publication}

Not applicable.

\section{Competing interests}

The authors declare that they have no competing interests.

\section{References}

1. Shah ASV, Sandoval Y, Noaman A, Sexter A, Vaswani A, Smith SW, Gibbins M, Griffiths M, Chapman AR, Strachan FE, et al: Patient selection for high sensitivity cardiac troponin testing and diagnosis of myocardial infarction: Prospective cohort study. BMJ 359: j4788, 2017.

2. Bressi E, Mangiacapra F, Ricottini E, Cavallari I, Colaiori I Di Gioia G, Creta A, Capuano M, Viscusi MM and Di Sciascio G: Impact of Neutrophil-to-Lymphocyte ratio and Platelet-to-lymphocyte ratio on 5-year clinical outcomes of patients with stable coronary artery disease undergoing elective percutaneous coronary intervention. J Cardiovasc Transl Res 11: 517-523, 2018.

3. Chen X, Shao M, Zhang T, Meng YB, Zhang W, Huang Z, Xu XG and $\mathrm{Li} \mathrm{GH}$ : Prognostic value of elevated mean platelet volume in acute myocardial infarction: A meta-analysis including 8,945 patients. Int J Clin Exp Med 12: 10151-10163, 2019. 
4. Turk J, Fourny M, Yayehd K, Picard N, Ageron FX, Boussat B, Belle L, Vanzetto $\mathrm{G}$, Puymirat $\mathrm{E}$, Labarère $\mathrm{J}$ and Debaty $\mathrm{G}$ : Age-related differences in reperfusion therapy and outcomes for ST-segment elevation myocardial infarction. J Am Geriatr Soc 66: 1325-1331, 2018.

5. Lin A, Devlin G, Lee M and Kerr AJ: Performance of the GRACE scores in a New Zealand acute coronary syndrome cohort. Heart 100: 1960-1966, 2014.

6. Shuvy M, Beeri G, Klein E, Cohen T, Shlomo N, Minha S and Pereg D: Accuracy of the global registry of acute coronary events (GRACE) risk score in contemporary treatment of patients with acute coronary syndrome. Can J Cardiol 34: 1613-1617, 2018.

7. Gray HH and Henderson RA: The GRACE score's performance in predicting in-hospital and 1-year outcome. Heart 97: 1461-1462, 2011.

8. Chen YH, Huang SS and Lin SJ: TIMI and GRACE risk scores predict both short-term and long-term outcomes in Chinese patients with acute myocardial infarction. Acta Cardiol Sin 34 4-12, 2018.

9. Jakimov T, Mrdović I, Filipović B, Zdravković M, Djoković A, Hinić S, Milić N and Filipović B: Comparison of RISK-PCI, GRACE, TIMI risk scores for prediction of major adverse cardiac events in patients with acute coronary syndrome. Croat Med J 58: 406-415, 2017.

10. Xiang L, Wang M, You T, Jiao Y, Chen J and Xu W: Prognostic value of ventricular wall motion score and global registry of acute coronary events score in patients with acute myocardial infarction. Am J Med Sci 354: 27-32, 2017.

11. González-Pacheco H, Bojalil R, Amezcua-Guerra LM, Sandoval J, Eid-Lidt G, Arias-Mendoza A, Azar-Manzur F, Álvarez-Sangabriel A, Altamirano-Castillo A, Briseño-Cruz JL, et al: Derivation and validation of a simple inflammation-based risk score system for predicting in-hospital mortality in acute coronary syndrome patients. J Cardiol 73 416-424, 2019

12. Wang JJ, Fan Y, Zhu Y, Zhang JD, Zhang SM, Wan ZF, Su HL and Jiang N: Biomarkers enhance the long-term predictive ability of the KAMIR risk score in Chinese patients with ST-elevation myocardial infarction. Chin Med J (Engl) 132: 30-41, 2019.

13. Ridker PM, Everett BM, Thuren T, MacFadyen JG, Chang WH Ballantyne C, Fonseca F, Nicolau J, Koenig W, Anker SD, et al: Antiinflammatory therapy with canakinumab for atherosclerotic disease. N Engl J Med 377: 1119-1131, 2017.

14. Fuentes QE, Fuentes QF, Andrés V, Pello OM, Font de Mora J and Palomo GI: Role of platelets as mediators that link inflammation and thrombosis in atherosclerosis. Platelets 24: 255-262, 2013.

15. Linden MD and Jackson DE: Platelets: Pleiotropic roles in atherogenesis and atherothrombosis. Int J Biochem Cell Biol 42 $1762-1766,2010$

16. Avci E, Kiris T, Çelik A, Variş E, Esin FK, Köprülü D and Kadi H: Prognostic value of rising mean platelet volume during hospitalization in patients with ST-segment elevation myocardia infarction treated with primary percutaneous coronary intervention. BMC Cardiovasc Disord 18: 226, 2018.

17. Sage AP and Mallat Z: Multiple potential roles for B cells in atherosclerosis. Ann Med 46: 297-303, 2014.

18. Moriya J: Critical roles of inflammation in atherosclerosis J Cardiol 73: 22-27, 2019.

19. Hudzik B, Szkodziński J, Lekston A, Gierlotka M, Poloński L and Gasior M: Mean platelet volume-to-lymphocyte ratio: A novel marker of poor short- and long-term prognosis in patients with diabetes mellitus and acute myocardial infarction. J Diabetes Complications 30: 1097-1102, 2016.

20. Kurtul A and Acikgoz SK: Usefulness of mean platelet Volume-to-Lymphocyte ratio for predicting angiographic No-Reflow and Short-Term prognosis after primary percutaneous coronary intervention in patients with ST-Segment elevation myocardial infarction. Am J Cardiol 120: 534-541, 2017.

21. O'Gara PT, Kushner FG, Ascheim DD, Casey DE Jr, Chung MK, de Lemos JA, Ettinger SM, Fang JC, Fesmire FM, Franklin BA, et al: 2013 ACCF/AHA guideline for the management of ST-elevation myocardial infarction: A report of the American College of Cardiology Foundation/American Heart Association task force on practice guidelines. Circulation 127: e362-e425, 2013

22. Maruhashi T, Ishihara M, Inoue I, Kawagoe T, Shimatani Y, Kurisu S, Nakama Y, Kagawa E, Dai K, Matsushita J and Ikenaga $\mathrm{H}$ : Effect of prodromal angina pectoris on the infarct progression in patients with first ST-elevation acute myocardial infarction. Circ J 74: 1651-1657, 2010.
23. Bayramoğlu A, Tasolar H, Kaya A, Tanboğa İH, Yaman M, Bektaş O, Günaydın ZY and Oduncu V: Prediction of no-reflow and major adverse cardiovascular events with a new scoring system in STEMI patients. J Interv Cardiol 31: 144-149, 2018.

24. Fan Z, Li Y, Ji H and Jian X: Prognostic utility of the combination of monocyte-to-lymphocyte ratio and neutrophil-to-lymphocyte ratio in patients with NSTEMI after primary percutaneous coronary intervention: A retrospective cohort study. BMJ Open 8 : $\mathrm{e} 023459,2018$

25. Shin HC, Jang JS, Jin HY, Seo JS, Yang TH, Kim DK and Kim DS: Combined use of neutrophil to lymphocyte ratio and C-reactive protein level to predict clinical outcomes in acute myocardial infarction patients undergoing percutaneous coronary intervention. Korean Circ J 47: 383-391, 2017.

26. Celik T, Kaya MG, Akpek M, Gunebakmaz O, Balta S, Sarli B, Duran M, Demirkol S, Uysal OK, Oguzhan A and Gibson CM: Predictive value of admission platelet volume indices for In-hospital major adverse cardiovascular events in acute ST-segment elevation myocardial infarction. Angiology 66: $155-162,2015$

27. Kırış T, Yazici S, Günaydin ZY, Akyüz Ş, Güzelburç Ö, Atmaca H, Ertürk M, Nazli C and Dogan A: The prognostic impact of In-hospital change in mean platelet volume in patients with Non-ST-Segment elevation myocardial infarction. Angiology 67: 690-696, 2016.

28. Machado GP, Araujo GN, Carpes CK, Lech M, Mariani S, Valle FH, Bergoli LCC, Gonçalves SC, Wainstein RV and Wainstein MV: Comparison of Neutrophil-to-Lymphocyte ratio and mean platelet volume in the prediction of adverse events after primary percutaneous coronary intervention in patients with ST-elevation myocardial infarction. Atherosclerosis 274: 212-217, 2018.

29. Monteiro Júnior JGM, Torres DOC, da Silva MCFC, Martins CMH, da Silva IK, do Nascimento MEM, Dos Santos ACO, Montarroyos UR and Filho DCS: Prognostic value of hematological parameters in patients with acute myocardial infarction: Intrahospital outcomes. PLoS One 13: e0194897, 2018.

30. Ranjith MP, DivyaRaj R, Mathew D, George B and Krishnan MN: Mean platelet volume and cardiovascular outcomes in acute myocardial infarction. Heart Asia 8: 16-20, 2016.

31. Wasilewski J, Desperak P, Hawranek M, Ciślak A, Osadnik T, Pyka Ł, Gawlita M, Bujak K, Niedziela J, Krawczyk M and Gąsior M: Prognostic implications of mean platelet volume on short- and long-term outcomes among patients with non-ST-segment elevation myocardial infarction treated with percutaneous coronary intervention: A single-center large observational study. Platelets 27: 452-458, 2016.

32. Goncalves SC, Labinaz M, Le May M, Glover C, Froeschl M, Marquis JF, O'Brien E, Shukla D, Ruchin P, Sookur D, et al: Usefulness of mean platelet volume as a biomarker for long-term outcomes after percutaneous coronary intervention. Am J Cardiol 107: 204-209, 2011

33. Núñez J, Miñana G, Bodí V, Núñez E, Sanchis J, Husser O and Llàcer A: Low lymphocyte count and cardiovascular diseases. Curr Med Chem 18: 3226-3233, 2011.

34. Wagdy S, Sobhy M and Loutfi M: Neutrophil/lymphocyte ratio as a predictor of In-hospital major adverse cardiac events, New-onset atrial fibrillation, and no-reflow phenomenon in patients with ST elevation myocardial infarction. Clin Med Insights Cardiol 10: 19-22, 2016.

35. Gok M, Kundi H, Kiziltunc E, Evlice M, Cetin M, Suleymanoglu M, Kurtul A and Ornek E: Relationship between prodromal angina pectoris and Neutrophil-to lymphocyte ratio in patients with ST elevation myocardial infarction. Heart Lung Circ 28: 901-907, 2019.

36. Heusch G and Gersh BJ: The pathophysiology of acute myocardial infarction and strategies of protection beyond reperfusion: A continual challenge. Eur Heart J 38: 774-784, 2017.

37. Orbegozo Cortés D, Su F, Santacruz C, Hosokawa K, Donadello K, Creteur J, De Backer D and Vincent JL: Ischemic conditioning protects the microcirculation, preserves organ function, and prolongs survival in sepsis. Shock 45: 419-427, 2016.

38. Zhang H, Qiu B, Zhang Y, Cao Y, Zhang X, Wu Z, Wang S and Mei L: The value of Pre-infarction angina and plasma D-Dimer in predicting No-reflow after primary percutaneous coronary intervention in ST-Segment elevation acute myocardial infarction patients. Med Sci Monit 24: 4528-4535, 2018. 
39. Kharbanda RK, Peters M, Walton B, Kattenhorn M, Mullen M, Klein N, Vallance P, Deanfield J and MacAllister R: Ischemic preconditioning prevents endothelial injury and systemic neutrophil activation during ischemia-reperfusion in humans in vivo. Circulation 103: 1624-1630, 2001.

40. Ornek E and Kurtul A: Relationship of mean platelet volume to lymphocyte ratio and coronary collateral circulation in patients with stable angina pectoris. Coron Artery Dis 28: 492-497, 2017.

41. Kilic A and Kurtul A: RETRACTED: Mean platelet Volume-to-Lymphocyte ratio as a novel marker for severity and complexity of coronary atherosclerosis in patients with acute coronary syndrome. Angiology: Jan 1, 2017 doi: 10.1177/0003319717724274 (Epub ahead of print).

42. Tang EW, Wong CK and Herbison P: Global registry of acute coronary events (GRACE) hospital discharge risk score accurately predicts long-term mortality post acute coronary syndrome. Am Heart J 153: 29-35, 2007.

43. Moady G, Iakobishvili Z, Beigel R, Shlomo N, Matetzky S, Zahger D and Atar S: The predictive value of low admission hemoglobin over the GRACE score in patients with acute coronary syndrome. J Cardiol 73: 271-275, 2019.

44. Zhang S, Wan Z, Zhang Y, Fan Y, Gu W, Li F, Meng L, Zeng X, Han D and Li X: Neutrophil count improves the GRACE risk score prediction of clinical outcomes in patients with ST-elevation myocardial infarction. Atherosclerosis 241: 723-728, 2015.
45. Klingenberg R, Aghlmandi S, Räber L, Gencer B, Nanchen D, Heg D, Carballo S, Rodondi N, Mach F, Windecker S, et al: Improved risk stratification of patients with acute coronary syndromes using a combination of hsTnT, NT-proBNP and hsCRP with the GRACE score. Eur Heart J Acute Cardiovasc Care 7: 129-138, 2018

46. Shahzad S, Mateen S, Hasan A and Moin S: GRACE score of myocardial infarction patients correlates with oxidative stress index, hsCRP and inflammation. Immunobiology 224: 433-439, 2019.

47. Fan Y, Wang J, Zhang S, Wan Z, Zhou D, Ding Y, He Q and Xie P: Homocysteine enhances the predictive value of the GRACE risk score in patients with ST-elevation myocardial infarction. Anatol J Cardiol 18: 182-193, 2017

48. Xiao L, Jia Y, Wang X and Huang H: The impact of preoperative fibrinogen-albumin ratio on mortality in patients with acute ST-segment elevation myocardial infarction undergoing primary percutaneous coronary intervention. Clin Chim Acta 493: 8-13, 2019. Attribution-NonCommercial-NoDerivatives 4.0 International (CC BY-NC-ND 4.0) License. 